Nota

\title{
Diminutivos atenuadores en narraciones de experiencia personal de hablantes de Santiago de Chile: ¡fenómeno variable?
}

\section{Mitigating diminutives in narratives of personal experiences from people in the city of Santiago de Chile: Is it a variable phenomenon?}

Javier Andrés González Riffo

Universidad Tecnológica de Chile INACAP

javier.gonzalez85@inacapmail.cl

Original recibido: 2018/09/09

Dictamen enviado al autor: 2019/05/30

Aceptado: 2019/09/23

\begin{abstract}
This study, which takes into account the mitigating side of diminutives, analyzes if there is a sociolinguistic variation in the Santiago de Chile variety regarding the use of mitigating diminutives in relation to factors such as sex, socioeconomic segment and type of narrative construction. In order to achieve this, the conceptual and methodological frameworks of variationist and interactional sociolinguistics were used. The analysis was carried out based on 90 narratives of personal experiences: 36 individual and 54 conversational. The study shows that diminutives are mainly used in male individual narratives, and in male-to-male conversational narratives belonging to a low socioeconomic segment. The lack of statistically-significant results may lead to reevaluate the
\end{abstract}

Cómo citar: González Riffo, Javier A., 2019. Diminutivos atenuadores en narraciones de experiencia personal de hablantes de Santiago de Chile: ¿fenómeno variable? Cuadernos de Lingüística de El Colegio de México 6(1). e152. DOI: 10.24201/clecm.v6i1.124. 
idea that it is a widespread phenomenon throughout the speech community being studied.

Keywords: variationist sociolinguistics, narratives, diminutives, modality, mitigation.

\section{Resumen}

Considerando la faceta atenuadora de los diminutivos, en este estudio se analiza si en la variedad de Santiago de Chile existe variación sociolingüística en el empleo de los diminutos atenuadores en relación con los factores sexo, grupo socioeconómico y tipo de construcción narrativa. Para esto, se utiliza el marco conceptual y metodológico de la sociolingüística variacionista. El análisis se realiza sobre la base de 90 narraciones de experiencia personal: 36 individuales y 54 conversacionales. Del estudio se desprende que los diminutivos se elicitan más en narraciones individuales de hombres, y hombre con hombre en relatos conversacionales del grupo socioeconómico bajo. La ausencia de resultados estadísticamente significativos podría replantear la idea de que se trata de un fenómeno extendido transversalmente en la comunidad de habla estudiada.

Palabras clave: sociolingüística variacionista, narraciones, diminutivos, modalidad, atenuación.

\section{INTRODUCCIÓN}

\subsection{Naturaleza, alcance y objetivos del estudio}

En el marco de la sociolingüística, los estudios sociopragmáticos han cautivado un importante interés en el último tiempo, en tanto que han per- 
mitido describir cómo los hablantes comunican y recrean sus propias identidades por medio de fenómenos que se suscitan en la interacción cotidiana. En este contexto, el desarrollo de la vertiente interaccional de la sociolingüística, con Gumperz (cf. 1971; 1982; 1999; 2001) como uno de sus precursores, ha aportado contundentemente con aquellos propósitos.

La sociolingüística interaccional, entre otras cosas, ha permitido añadir metodologías de corte cualitativo a los estudios del lenguaje en su contexto social, que tienen como propósito ampliar la mirada que el variacionismo ponía a disposición para observar al lenguaje contextualizado. Además, ha facilitado la incorporación de factores externos que a la entrevista sociolingüística tradicional le dificultaba considerar, tales como la distancia social de los hablantes. Como es bien sabido, la entrevista sociolingüística ha motivado cuestionamientos hacia el método variacionista, en particular porque no daría cuenta del contexto interaccional tal como ocurre en la comunicación cotidiana (Norrick 2000; Thornborrow 2012). En este contexto, la inclusión de materiales elicitados a partir de diferentes enfoques podría aportar en la discusión tanto teórico-conceptual como también metodológica. Consiguientemente, en esta investigación se trabaja con dos tipos de narrativas: las individuales, provenientes de entrevistas semiestructuradas en el sentido clásico, y las conversacionales (interaccionales, coconstruidas o diádicas) construidas mediante las directrices metodológicas del 'diseño narrativo' cualitativo.

Los estudios sociolingüísticos han permitido, entre otras cosas, confirmar o derribar estereotipos sociolingüísticos; esto es, ideas que se han instalado sobre la forma en que los hablantes se comunican. Entre los 
estereotipos, se cuenta con que tanto en Chile como fuera del país se ha expandido la idea de que el chileno habla en chiquitito, idea que incluso se ha trabajado en diversos artículos de prensa (cf. Navarrete, 2014; Errázuriz, 2014). En esta línea, y considerando la faceta atenuadora de este recurso lingüístico, cabe preguntarse si en la variedad chilena el uso de diminutivo se comportará o no como un fenómeno variable. En este contexto, en este estudio se analiza el uso de diminutivos a partir de los presupuestos teórico-metodológicos de la sociolingüística variacionista.

Los diminutivos pueden constituir un recurso que sirve para la atenuación lingüística en el caso de las narraciones de experiencia personal, tanto para las construidas individualmente como para las conversacionales por dos hablantes en un contexto de interacción (González Riffo \& Guerrero González 2017). En concreto, la utilización de atenuadores discursivos permite a los hablantes estratégicamente cuidar su propia imagen y las de otros en la interacción, en los mismos términos de Goffman (1959; 1967; 1974; 1981), en beneficio de la armonía interpersonal. En consecuencia, los objetivos específicos que direccionan esta pesquisa son (1) comparar el uso de este recurso en el total de narraciones individuales y diádicas; y (2) comprobar si existe variación según el tipo de construcción discursiva en relación con los factores sexo y el grupo socioeconómico de los hablantes.

$\mathrm{El}$ análisis se realiza sobre la base de un total de 36 narraciones individuales y 54 narraciones conversacionales. Todos los hablantes tienen edades que oscilan entre los 35 y los 54 años, etapa vital que se caracteriza por la plenitud del desarrollo laboral y profesional de los individuos (Blas Arroyo 2005) y que, para el caso del español de Chile, ha demos- 
trado ser el grupo etario más sensible a la variación (Guerrero González 2014). Los resultados de esta pesquisa permitirán resolver si, pese a la aparente extensión del recurso en la comunidad de habla, existe o no variación sociolingüística.

\section{Marco conceptual}

\subsection{Atenuación y variación sociolingüística}

La atenuación constituye, según Montecino (2003), una categoría pragmática junto con la intensificación. A juicio de Briz (2007), se trata de una estrategia de distancia lingüística que utilizan los hablantes para conseguir un acercamiento social con otros sujetos por medio del distanciamiento de aquello que se dice. La dualidad entre atenuación e intensificación, en cambio, puede ser desechada si se considera que la atenuación es una estrategia con la cual los hablantes realizan procedimientos de distinta naturaleza por medio del lenguaje más bien para direccionar los efectos perlocutivos de sus actos de habla en beneficio de la imagen de los interactuantes y, consecutivamente, de la armonía interpersonal (González Riffo \& Guerrero González 2017), relegando al carácter semántico de la atenuación a un concepto más bien de matiz y abriendo la posibilidad de considerar la atenuación y la intensificación no como un continuo, sino como fenómenos que se interrelacionan en beneficio del compromiso discursivo por medio del concepto de modalidad, en la línea que propone Monjour (2018). 
Briz \& Albelda (2013) han dilucidado que la atenuación es un fenómeno que varía según el contexto situacional de la interacción. En concreto, hay factores interpersonales, genéricos y pragmáticos que pueden propiciar el mayor o menor uso de atenuadores en el discurso, por lo que se puede proponer un continuo que comprende dos ejes: la coloquialidad y mayor inmediatez comunicativa, por un lado, y la formalidad y menor inmediatez comunicativa, por el otro. Sería en este último eje, según los investigadores, en el que se presentaría mayor frecuencia en el uso de atenuadores.

Sin embargo, investigaciones como la de Cestero \& Albelda (2012) han permitido ampliar el carácter variable de la atenuación no solo a lo situacional, sino también a lo social. El mayor o menor uso de atenuadores revelaría también cómo los hablantes, insertos en sus comunidades de habla, adquieren patrones de comportamiento según distintos factores sociales que ilustrarían cómo los individuos construyen y negocian sus propias identidades. Así, los aportes de Cestero (2011) y de Molina Martos (2015) en Madrid y en el barrio de Salamanca, respectivamente, son capitales para demostrar la variación sociolingüística del fenómeno en tanto comprueban la correlación entre el uso de atenuadores y factores sociales tales como el sexo y el nivel de instrucción de los hablantes.

\subsubsection{La atenuación en el marco de las narraciones de experiencia personal}

Los estudios sobre atenuación se han realizado en una amplia gama de géneros discursivos, entre ellos, prensa (cf. Bouzas 2017), comentarios 
en línea (cf. Moya \& Carrió-Pastor 2018), discurso político (cf. Infante \& Flores 2017; Estrada \& Zárate 2017) y discurso argumentativo en general (cf. González Riffo \& Guerrero González 2017; Rodríguez Alfano 2018). Desde una perspectiva sociolingüística, las investigaciones han empleado la entrevista sociolingüística como corpus de análisis, sin reparar en los distintos tipos de discursos que en ella surgen. ${ }^{1}$ En particular, la atenuación en narraciones de experiencia personal ha sido escasamente revisada.

Las narraciones de experiencia personal son definidas por Labov (1972) como un método de recapitulación de experiencias pasadas que se expresan por medio de una secuencia verbal de proposiciones. Estas últimas se instancian discursivamente por medio de cláusulas que se organizarían "en el orden en que el evento original ocurrió" (Labov 2013: 15, traducción propia). Tales secuencias se organizan en una macroestructura que, para Labov \& Waletzky (1967), se compone de seis rasgos estructurales: el resumen, la orientación, la acción de complicación, la evaluación, el resultado o resolución y la coda, aunque la posición de cada elemento dentro del relato, así como también su presencia, es variable (Guerrero González 2017).

Ochs (2004) caracteriza las narraciones desde su función referencial y desde su función expresiva. Esto último tiene su origen en el concepto de 'reportabilidad': para Labov (2013) un elemento crucial de las narraciones para ser contadas, por una parte, y estudiadas, por otra, es que

1 La entrevista sociolingüística permite elicitar diferentes tipos de discurso, entre ellos, expositivo, narrativo y argumentativo. 
deben ser dignas de ser contadas. Sin embargo, según el mismo autor, la reportabilidad está inversamente relacionada con la credibilidad; es decir, mientras más sorprendentes sean las historias, serán más dignas de ser contadas, pero lo increíble disminuye la credibilidad de las mismas. Por esto, el narrador debe evaluar constantemente su relato con el fin de validarlo y, así, no dañar su estatus.

Trabajando con "el principio de que el narrador no miente, por lo que utiliza un amplio repertorio de recursos para transformar la realidad sin mentir" (Labov 2013: 10, traducción propia), este estudio parte de la premisa de que la atenuación responde a un mecanismo de evaluación para mantener tanto la reportabilidad como la credibilidad sin afectar la imagen del narrador. Esto se debería a que los hablantes buscan la armonía interpersonal en la interacción, lo que se relaciona en parte con el compromiso discursivo (González Riffo \& Guerrero González 2017).

\subsection{Caracterización gramatical y pragmática de los diminutivos}

En términos morfológicos, en español, los diminutivos corresponden a distintos sufijos entre los cuales se encuentran -ito/-ita, -ico/-ica e -illo/illa, entre otros, junto con sus posibles flexiones de género. En este contexto, el Manual de la Nueva Gramática de la Lengua Española (2010) considera a este tipo de sufijos como uno de tipo apreciativo, es decir, aquellos que se caracterizan por "expresar la valoración afectiva que se hace de las personas o las cosas" (163). Asimismo, reconoce tres posibles interpretaciones que se puede hacer de este: el de tamaño reducido (inclusión del sema +Pequeño o, simplemente, de disminución semánti- 
ca), la de intensificación (cuando se combinan con adjetivos o adverbios, como en bien rapidito) y la de atenuación. Sobre esta compleja naturaleza de los diminutivos ha habido interesantes debates al respecto (cf. Hummel 1997).

Diversas sistematizaciones de recursos de atenuación para el español reconocen los diminutivos como una estrategia atenuadora. Por ejemplo, Briz \& Albelda (2013) y Albelda et al. (2014) los incorporan dentro de su taxonomía reparando los primeros en la potencialidad de que no todo sufijo apreciativo es atenuante. En esta misma línea se encuentra la propuesta de Cestero \& Rodríguez Alfano (2014) para el estudio de la atenuación en el corpus PRESEEA. En estas propuestas se asume que los atenuadores -y, por consiguiente, los diminutivos- pueden desempeñar tres funciones que los hace ser, justamente, atenuadores: la salvaguarda de la propia imagen, la prevención de posibles amenazas a la imagen y la reparación de una amenaza a la imagen o al territorio. ${ }^{2}$ Puga (1997) coincide en que los diminutivos, para el caso del español de Chile, representan un recurso de atenuación. Haverkate (1994) también destaca el valor atenuador de los diminutivos. La relación entre cortesía y diminutivos también ha sido destacada por Mendoza (2005) y Mariottini (2006). En este último trabajo se ha descrito cómo en los chats el uso de diminutivos generaría mayor cercanía interpersonal.

2 Siguiendo a Goffman (1959; 1967; 1974; 1981), la imagen -o face-corresponde a la visión que uno mismo y otros tienen de una persona. El territorio, por su parte, se relaciona con la libertad de acción de los sujetos. Ambos conceptos, como se ha de inferir, están estrechamente ligados. Estos postulados son fundamentales para los desarrollos de las teorías de cortesía postulados, entre otros, por Brown y Levinson (1987) y Leech (1983; 2014). 
En González Riffo \& Guerrero González (2017) se propone una clasificación de recursos de atenuación para el caso de la variedad chilena del español. Esta clasificación está pensada precisamente para el género narración de experiencia personal conversacional y considera como un aporte previo los trabajos de Briz \& Albelda (2013) y de Cestero \& Rodríguez Alfano (2014). En esta línea, los diminutivos corresponden a un recurso atenuador del eje pragmático denominado Estima, en que se sitúan aquellas estrategias que atienden al carácter afectivo de la interacción, pues los diminutivos agregarían un valor pragmático valorativo y no solo evaluativo -en términos de pequeñez-. Esto último incide en que se crea que se puede incluir al diminutivo que agrega el valor +pequeńo dentro de la categoría de atenuador siempre y cuando no se considere la imagen de los interlocutores como el elemento que únicamente designa si un recurso es o no atenuador, sino más bien la idea de compromiso discursivo, entendido como la empática predisposición de los interlocutores para ser parte de la interacción, lo que se vislumbra por medio del discurso (González Riffo 2017). ${ }^{3}$ Esto, debido, principalmente, a que el carácter evaluativo no es excluyente del valorativo. ${ }^{4}$

3 Otros conceptos similares, aunque con matices, son conversational engagement (Goodwin 1981), mutual engagement (Merritt 1982) e involvement (Gumperz 1982; Chafe 1985; Tannen 2007).

${ }^{4}$ Piénsese, por ejemplo, en el uso de un sustantivo propio con un diminutivo como $<$ Pablito $>$ para hacer referencia a un sujeto de nombre $<$ Pablo $>$. En este caso, la inclusión del diminutivo no necesariamente implica que Pablo sea de baja estatura, sino que puede haber un componente afectivo que motiva la inclusión del sufijo. De la misma forma, aunque el referente designado en efecto sea de baja estatura, el sistema de la lengua ofrece variadas formas para 
Así, el carácter $\operatorname{modal}^{5}$ del diminutivo permite entender que la inclusión de afecto atenúa porque en la narración persuade emotivamente al interlocutor a no poner en duda la credibilidad de la historia. Esto, en el contexto de la búsqueda de equilibrio entre credibilidad y reportabilidad (Labov 2013), presente en el género narración.

En este estudio se trabaja con la propuesta de González Riffo \& Guerrero González (2017), en que se sostiene que el diminutivo adquiere un carácter afectivo que atenúa al buscar empatía con el interlocutor, quien a su vez evalúa la credibilidad de la historia narrada. En el siguiente ejemplo se puede observar tal carácter afectivo:

(1) y Jesús se fue en ese momento // se fue // y yo lo miraba / y yo sabía que él me estaba escuchando / y si yo lo hablaba /en el pensamiento él

expresar ese contenido (como <El pequeño Pablo $>$ ), por lo que es posible pensar que, independiente de las características particulares del referente, la inclusión del diminutivo siempre podría cargar con un carácter afectivo.

5 Monjour (2018) propone que, en ciertos géneros, la atenuación más bien se relaciona con el compromiso de los hablantes al igual que la intensificación, no estableciendo un continuum en que ambos fenómenos se contraponen, sino que más bien se complementan. Para ello, el autor propone aunar ambos fenómenos bajo el rótulo de la modalidad. En este sentido, y dadas las características de la narración, se cree que bajo la premisa anteriormente señalada el valor afectivo sí atenúa, pues pragmáticamente permite persuadir o disuadir a los interlocutores de ciertas actitudes que cuestionen la imagen del narrador, lo que concierne a una prevención ante el ataque de la imagen propia, en los términos de Albelda et al. (2014). Un lineamiento similar indica Malaver (2018) en su estudio sociolingüístico del diminutivo en el español de Caracas, pues considera que aquel valor subjetivo del diminutivo también puede atenuar. En lo que respecta a la modalidad, en especial a la apreciativa, es esencial el aporte de Gosselin (2010). 
me escucha / entonces yo lo miraba por detrás / y le veía su pelito largo bonito / y lo miraba / y le hablaba en el pensamiento // y le decía Señor / sí la televisión a mí me la regalaron y yo le miraba a los discípulos que están ahí / y les decía pero sí / sí es verdad / sí me la regalaron. (SCHI_H11_021)

En (1), el hablante es consultado por algún sueño premonitorio. En ese contexto, relata un sueño en que tuvo una conversación con Jesús, quien ponía en duda cómo el hablante había conseguido su televisor. Aquí, se ve enfrentado a una situación compleja, que es justamente la de relatar un sueño en que aparece un ser divino propio de ciertas creencias religiosas y, en consecuencia, cuestionable para quienes no comparten los mismos dogmas. Ante eso, el narrador utiliza diminutivos en una cláusula que, además, presenta una valoración positiva inscrita que atañe justamente a ese individuo. Siguiendo el razonamiento de González Riffo \& Guerrero González (2017), en la tarea de probar que la historia es verdadera, pese a que es poco creíble, los narradores utilizan recursos que generan empatía con sus interlocutores para que estos no enjuicien ni la historia ni a quien la relata; es decir, prevendrían ataques a su propia imagen. El carácter afectivo del diminutivo, en consecuencia, serviría como herramienta de persuasión. 
3. Metodología ${ }^{6}$

\subsection{Corpus}

Para el estudio se analizaron 90 narraciones de experiencia personal: 36 construidas individualmente y 54 conversacionales. Las primeras forman parte del corpus de entrevistas sociolingüísticas del Proyecto para el Estudio del Español de España y América de Santiago de Chile, PreseEa-sa, y las segundas del Proyecto Fondecyt 11150007.

El corpus del PRESEEA-SA corresponde a la reunión de entrevistas realizadas por estudiantes de las licenciaturas en Lengua y Literatura Hispánicas e Inglesas de la Universidad de Chile, cuyos informantes son hablantes santiaguinos de diversas edades, sexo y nivel de instrucción. De estas, únicamente se ha analizado la narración mejor desarrollada por hablantes con edades que oscilan entre los 35 y los 54 años. Tales narraciones poseen mayor cantidad de cláusulas narrativas y cumplen la estructura básica descrita por Labov \& Waletzky (1967) y que se han referenciado en el 2.1.1. Esto, con el propósito de neutralizar variables intervinientes tales como el cansancio y que, de esta forma, las narraciones fueran contrastables entre sujetos.

Por su parte, el corpus del Proyecto fONDECYT 1150007 se compone de 54 narraciones conversacionales videograbadas, esto es, construidas por dos hablantes que narran una historia que vivieron juntos. Todos

${ }^{6}$ La metodología aquí presentada replica la de las investigaciones derivadas del proyecto FONDECYT 11150007, particularmente el análisis inductivo de las narraciones conversacionales. 
los informantes al momento de participar tienen edades del grupo etario previamente mencionado. Además, el corpus se organiza según el sexo de los participantes (hombre-hombre, mujer-hombre y mujer-mujer) y su grupo socioeconómico. Las narraciones se elicitan por medio del diseño narrativo para el estudio autobiográfico y de tópico.

Cabe destacar que en este estudio se utiliza la variable grupo socioeconómico y no la de nivel de instrucción. Esto, porque se ha complementado la muestra de PRESEEA con la metodología de $\operatorname{ESECH}^{7}$ para hacer ambos corpus comparables. En concreto, el método considera el nivel de instrucción como el más relevante para la categorización de un grupo socioeconómico.

\subsection{Población y muestra}

La muestra recoge narraciones de hablantes santiaguinos (cf. Prieto 19951996) cuyas edades oscilan entre los 35 y los 54 años. Siguiendo a Blas Arroyo (2005), se trataría de hablantes que coinciden en la etapa vital en que los sujetos se desarrollan laboralmente de forma plena y que, además, han demostrado ser el grupo más sensible a la variación en la variedad santiaguina (Guerrero González 2014). La muestra se organizó en un sistema de cuotas con afijación uniforme (López Morales 1994), tal y como se ilustra en las siguientes tablas:

7 Proyecto de Estudio Sociolingüístico del Español de Chile. 
Tabla 1. Tabla de distribución de los informantes en el corpus de narraciones individuales

\begin{tabular}{lccc}
\hline $\begin{array}{l}\text { Grupo } \\
\text { socioeconómico }\end{array}$ & $\begin{array}{c}\text { Hombres } \\
34-54 \text { años }\end{array}$ & $\begin{array}{c}\text { Mujeres } \\
34-54 \text { años }\end{array}$ & Totales \\
\hline Bajo (B) & 6 & 6 & 12 \\
Medio (M) & 6 & 6 & 12 \\
Alto (A) & 6 & 6 & 12 \\
Totales & 18 & 18 & 36 \\
\hline
\end{tabular}

Tabla 2. Tabla de distribución de los informantes en el corpus de narraciones conversacionales

\begin{tabular}{lcccc}
\hline $\begin{array}{l}\text { Grupo } \\
\text { socioeconómico }\end{array}$ & $\begin{array}{c}\text { Hombre-Hombre } \\
(\mathrm{H}-\mathrm{H})\end{array}$ & $\begin{array}{c}\text { Hombre-Mujer } \\
(\mathrm{H}-\mathrm{M})\end{array}$ & $\begin{array}{c}\text { Mujer-Mujer } \\
(\mathrm{M}-\mathrm{M})\end{array}$ & Totales \\
\hline Bajo (B) & 6 & 6 & 6 & 18 \\
Medio (M) & 6 & 6 & 6 & 18 \\
Alto (A) & 6 & 6 & 6 & 18 \\
Totales & 18 & 18 & 18 & 54 \\
\hline
\end{tabular}

\subsection{Procedimiento analitico}

Posterior a la selección de las secuencias a analizar, primero se determina la frecuencia de empleo en cada narración y en consideración del total de cláusulas que la componen, lo que luego se correlaciona con los factores en estudio: tipo de construcción, sexo y grupo socioeconómico. Cabe destacar que varias propuestas para el estudio sociolingüístico de la atenuación, entre ellas las declaradas en $\$ 2.2$, realizan el análisis sobre 
la cantidad de atenuadores por acto de habla. Esto se debe, principalmente, a que utilizan la entrevista sociolingüística casi en su totalidad, habiendo, entonces, turnos de habla que suelen ser breves. La narración, por su parte, suele plantearse en uno o más turnos de habla mucho más extensos. Por esto, y también con la finalidad de constatar la frecuencia de empleo de los atenuadores en unidades de sentido, se utiliza la cláusula como unidad de análisis, entendida esta como una estructura en que se combinan tres tipos de significados: ideacional, interpersonal y textual (Halliday 1985).

Para el procesamiento de los datos, se ha utilizado estadística descriptiva e inferencial. En lo que concierne a esta última, se utilizó el software de análisis estadístico R (R Core Team 2015) y se realizaron pruebas de Anova factorial entre sujetos considerando como variable dependiente la ocurrencia de diminutivos atenuadores promedio por cláusula. El uso de estas pruebas permitiría conocer si los resultados se deben o no al azar y, consecutivamente, si son expandibles a la comunidad de habla. Tales pruebas se efectuaron sobre ambos tipos de narraciones. Las variables independientes fueron el sexo y el grupo socioeconómico de los informantes y el tipo de construcción de la narración (individual o conversacional). Se consideró como estadísticamente significativos aquellos resultados en los que $\mathrm{p}<0.05$, por lo que se sigue las recomendaciones de Moreno Fernández (1990), López Morales (1994) y Hernández Campoy \& Almeida (2005). 


\section{Presentación y anÁlisis de los Resultados}

El uso de diminutivos, al conllevar una carga semántica afectiva, busca empatizar con su interlocutor: sea el entrevistador, en caso de una narración elicitada mediante entrevista sociolingüística, o a su compañero (o conarrador) y al entrevistador en el caso de las narraciones diádicas. Esta búsqueda por empatizar con el interlocutor se realizaría para persuadir al interlocutor de forma tal que la imagen propia o de otro no se vea amenazada -esto es: prevención de posibles amenazas a la imagen (Albelda $e t$ al. 2014), razón por la cual el diminutivo adquiere un valor de atenuador. A continuación, se presentan ejemplos hallados en el corpus.

(2) la T es especial / eeh / de todos los hermanos / de los seis hermanos que tiene / los cuatro / la T es diferente a todos // en cuanto a carińo / que no me deje sola // ella anda preocupada a pesar de que es tan chiquitita / anda preocupada / de hartas cosas que su hermana mayor no lo hace / pero ella sí (SCHI_M21_023)

(3) si bien / la muerte para los padres es traumática la muerte de un hijo // es lo más doloroso que / que uno puede experimentar sobre todo como madre porque uno que lo lleva / durante nueve meses en su guatita (H-M04B)

(4) y esto era todas las mañanas y yo me sentía feliz viendo eso po y y supe después que se trataba de unos ratoncitos silvestres (M-M06A)

En (2), la hablante enuncia esta expresión para justificar el comportamiento de su hija, quien, pese a su corta edad, se preocupa por su madre 
incluso más que su hermana mayor. Pese a que esta preocupación podría ser enjuiciable, cuestión que se observa en el uso del concesivo "a pesar de que", pues aparentemente no es una conducta esperada para la edad de la niña, la narradora lo destaca como algo positivo, lo que está en línea con las apreciaciones valorativas como "es especial" y la cuantificación de los hermanos que utiliza a modo de comparación. Aquella imagen positiva que se destaca de la niña también se vislumbra en el uso del diminutivo "chiquitita", cuyo valor afectivo promueve que el interlocutor justifique su actuar y, en consecuencia, no ponga en duda el relato.

En (3), el diminutivo "guatita" aparece dentro de otro recurso de atenuación: la justificación/explicación. Efectivamente, en el ejemplo citado se argumenta la idea de que la muerte de un hijo es una de las experiencias más dolorosas que alguien podría vivir, incluso más que la muerte de un padre. La razón por la cual la hablante cree aquello es porque una madre gesta a su hijo, situación que, arguye, establecería un vínculo afectivo muy importante. El carácter afectivo de "guatita" sirve como forma de 'intensificar' esa misma atenuación proferida ya por la justificación, lo que va también en línea con la idea de que los recursos de atenuación se disponen de forma solidaria, en tanto no suelen aparecer aislados en el discurso (Villalba 2018). La imagen de la narradora se ve amenazada en tanto la emisión de una opinión puede o no ser compartida por su interlocutor, razón por la cual las justificaciones previenen un potencial ataque a la imagen de la misma forma que lo hace el diminutivo.

El ejemplo (4) nuevamente relaciona el uso de diminutivo con la justificación y la explicación en general. Nótese que la informante, narrando una anécdota de sus vacaciones, comenta que era feliz viendo algo 
que resultó ser ratones silvestres. El diminutivo se puede interpretar a la luz de dos elementos: como una suerte de intensificación del carácter de felicidad y también para otorgarle un carácter de ternura al animal referido, configurando una suerte de contraexpectativa entendiendo que los ratones pueden ser asociados a otras reacciones que no necesariamente se vinculan con la felicidad. De esta manera, la inclusión del diminutivo permite salvaguardar la imagen de la informante, pues parecería entendible que le produzca felicidad el ver animales pequeños y tiernos. Así, se aminora la posibilidad de que existan posiciones adversas entre los narradores y con el entrevistador.

En total, en los 90 discursos analizados se identificaron 59 ocurrencias del fenómeno en estudio. De estas, 21 se hallaron en las narraciones individuales y 38 en las conversacionales. Pese a que evidentemente en estas últimas el fenómeno se halló más veces, al considerar la división por cláusulas, la media del índice de ocurrencia es de 1.26 diminutivos por cláusula en las narraciones individuales y 0.01 en las conversacionales. La diferencia se debe a que en las narraciones conversaciones hay una mayor cantidad de cláusulas por cada relato. Este índice permite revisar si el discurso se encuentra más o menos atenuado.

La Tabla 3 sintetiza y organiza los resultados recién señalados.

En lo que respecta a esta diferencia, cabría señalar que en las narraciones individuales se atenúa más por medio del uso de diminutivos que en las narraciones conversacionales; sin embargo, el dato no es estadísticamente significativo $(\mathrm{p}=0.1)$. Pese a esto, es relevante destacar que aquella tendencia sería transversal al general de los recursos de atenuación para las narraciones de experiencia personal (González Riffo 2017), lo que 
Tabla 3. Síntesis de frecuencias absolutas y medias asociadas a la relación entre uso de diminutivos y factor tipo de construcción de la narración

\begin{tabular}{lccccc}
\hline Factor & & $\begin{array}{c}\text { Total de } \\
\text { diminutivos }\end{array}$ & $\begin{array}{c}\text { Media de } \\
\text { diminutivos }\end{array}$ & $\begin{array}{c}\text { Media de } \\
\text { cláusulas }\end{array}$ & $\begin{array}{c}\text { Media } \\
\text { diminutivos/ } \\
\text { cláusulas }\end{array}$ \\
\hline \multirow{2}{*}{$\begin{array}{l}\text { Tipo de } \\
\text { construcción }\end{array}$} & $\begin{array}{c}\text { Narraciones } \\
\text { individuales }\end{array}$ & $21(35.5 \%)$ & 0.58 & 23.5 & 0.03 \\
& $\begin{array}{c}\text { Narraciones } \\
\text { conversacionales }\end{array}$ & $38(64.5 \%)$ & 0.7 & 64.3 & 0.014 \\
\hline
\end{tabular}

se podría deber a que el contexto de interacción de la entrevista sociolingüística incitaría al informante a proteger su propia imagen más que en un contexto en que el informante cuenta con otro una historia que ambos han vivido, pues, en el contexto de la pugna entre la credibilidad y la reportabilidad, la presencia de un conarrador que también vivió la historia podría ser argumento suficiente para dar la historia por verdadera. En este sentido, la presencia de un conarrador entregaría la suficiente consistencia argumentativa para asegurar la veracidad de la historia.

En lo que concierne al factor grupo socioeconómico, la Tabla 4 sintetiza los hallazgos y el Gráfico 1 los ilustra.

Del gráfico precedente, se observa que en el total de las narraciones y en las individuales son los hablantes de los grupos socioeconómicos bajos quienes utilizan más este recurso, lo que no es apreciable en las narraciones conversacionales. Frente a esto, se ha de destacar que los datos no son estadísticamente significativos (para las narraciones individuales: $\mathrm{p}=0.1$; para las conversacionales: $\mathrm{p}=0.3$; para el total de las narraciones: $p=0.3)$. Si bien se podría sostener que esto de igual forma contrasta 
Tabla 4. Síntesis de frecuencias absolutas y medias asociadas a la relación entre uso de diminutivos y factor grupo socioeconómico

\begin{tabular}{|c|c|c|c|c|c|}
\hline Factor & $\begin{array}{c}\text { Grupo } \\
\text { socio- } \\
\text { económico }\end{array}$ & $\begin{array}{c}\text { Total de } \\
\text { diminutivos }\end{array}$ & $\begin{array}{c}\text { Media de } \\
\text { diminutivos }\end{array}$ & $\begin{array}{l}\text { Media de } \\
\text { cláusulas }\end{array}$ & $\begin{array}{c}\text { Media } \\
\text { diminutivos/ } \\
\text { cláusulas }\end{array}$ \\
\hline \multirow{3}{*}{$\begin{array}{l}\text { Narraciones } \\
\text { individuales }\end{array}$} & Bajo & $13(61.9 \%)$ & 1.08 & 16.4 & 0.08 \\
\hline & Medio & $6(25.5 \%)$ & 0.5 & 32.8 & 0.01 \\
\hline & Alto & $2(12.6 \%)$ & 0.16 & 21.4 & 0.009 \\
\hline \multirow{3}{*}{$\begin{array}{l}\text { Narraciones } \\
\text { conversacionales }\end{array}$} & Bajo & $5(13.1 \%)$ & 0.27 & 49 & 0.004 \\
\hline & Medio & $12(31.5 \%)$ & 0.66 & 76.6 & 0.01 \\
\hline & Alto & $21(55.4 \%)$ & 1.16 & 67.4 & 0.01 \\
\hline \multirow{3}{*}{$\begin{array}{l}\text { Total de } \\
\text { narraciones }\end{array}$} & Bajo & $18(30.5 \%)$ & 0.6 & 36 & 0.03 \\
\hline & Medio & $18(30.5 \%)$ & 0.6 & 59.1 & 0.01 \\
\hline & Alto & $23(39 \%)$ & 0.76 & 49 & 0.01 \\
\hline
\end{tabular}

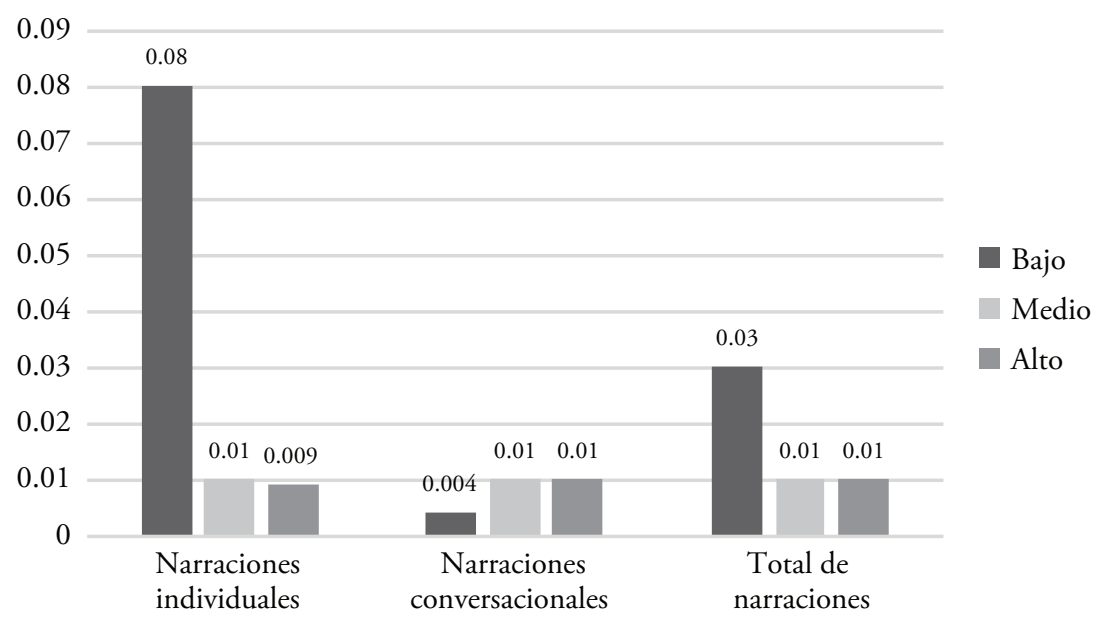

Gráfico 1. Promedio de diminutivos por cláusula en relación con el grupo socioeconómico de los informantes 
con la idea de que son los grupos socioeconómicos altos los que más atenúan (cf. Cestero 2011), es relevante recordar que los diminutivos constituyen solo uno de los tantos recursos que sirven para ese fin, por lo que aquella idea puede ser cierta sin desmedro del panorama que se presenta de este recurso.

Finalmente, concerniente al factor sexo, los datos nuevamente no son estadísticamente significativos ni en las narraciones individuales $(\mathrm{p}=0.4)$ ni en las conversacionales $(\mathrm{p}=0.3)$. En las primeras, se observa que son los hombres quienes más ocupan este recurso con una media de 0.04 frente a una de 0.02 de las mujeres. En las segundas, se observa que las narraciones de mujeres (media de 0.02) superan ampliamente las de hombres (media de 0.0099) y las mixtas (media de 0.0094) en su frecuencia de empleo. Estos datos se presentan en la Tabla 5.

Como el sexo es un factor que no es posible de comparar entre narraciones individuales y conversacionales, dado que en las segundas se trabaja con tres grupos dependiendo de los sexos de los informantes de cada narración diádica, se procede a continuación a revisar en primera instancia los resultados asociados a las primeras. El Gráfico 2 ilustra los resultados.

De las narraciones individuales, se observa que los hombres utilizan este recurso más que las mujeres, lo que coincidiría con el comportamiento atenuador de los recursos en general del barrio de Salamanca (cf. Molina Martos 2005) y Madrid (cf. Cestero 2011). Sin embargo, se observa una situación distinta en las narraciones conversacionales, como se indica en el Gráfico 3. 
Tabla 5. Síntesis de frecuencias absolutas y medias asociadas a la relación entre uso de diminutivos y factor sexo

\begin{tabular}{llcccc}
\hline Factor & & $\begin{array}{c}\text { Total de } \\
\text { diminutivos }\end{array}$ & $\begin{array}{c}\text { Media de } \\
\text { diminutivos }\end{array}$ & $\begin{array}{c}\text { Media de } \\
\text { cláusulas }\end{array}$ & $\begin{array}{c}\text { diminutivos/ } \\
\text { cláusulas }\end{array}$ \\
\hline Narraciones & Hombres & 15 & 0.83 & 28.8 & 0.04 \\
individuales & Mujeres & 6 & 0.33 & 18.2 & 0.02 \\
\hline \multirow{2}{*}{$\begin{array}{l}\text { Narraciones } \\
\text { conversacionales }\end{array}$} & Hombre-Hombre & 10 & 0.55 & 64.5 & 0.009 \\
& Hombre-Mujer & 11 & 0.61 & 76.3 & 0.009 \\
& Mujer-Mujer & 17 & 0.94 & 52.2 & 0.023 \\
\hline
\end{tabular}

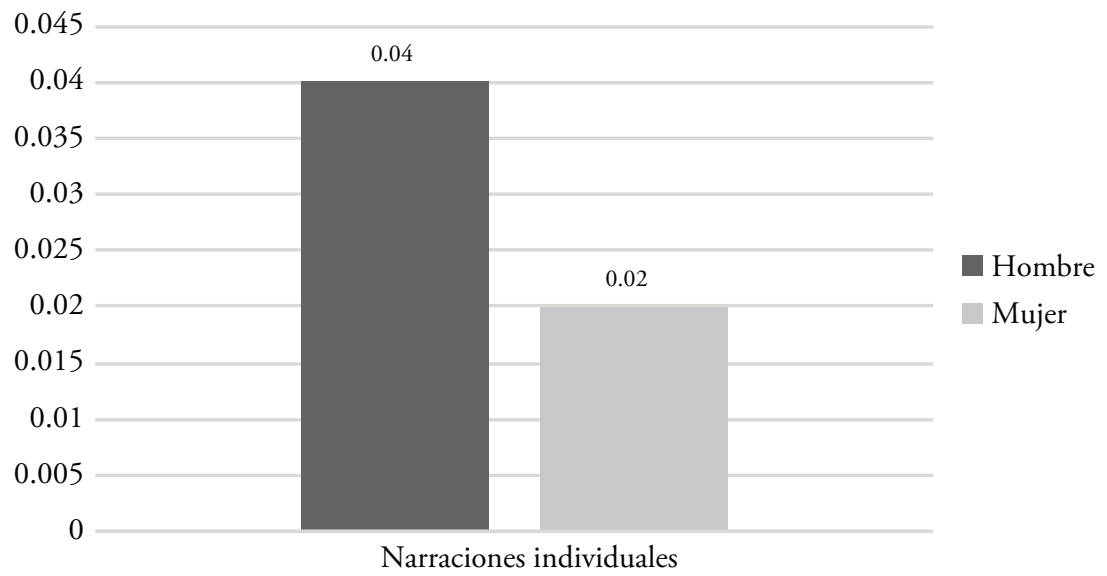

Gráfico 2. Promedio de diminutivos por cláusula en relación con el sexo del informante en narraciones individuales 


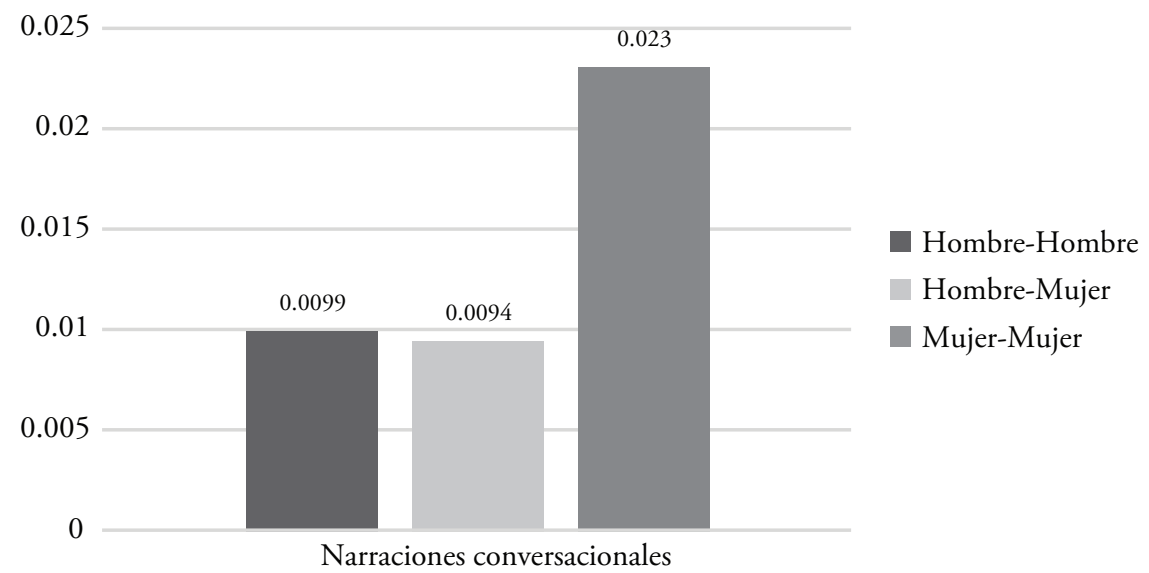

Gráfico 3. Promedio de diminutivos por cláusula en relación con el sexo de los informantes en narraciones conversacionales

Para el caso de las narraciones conversacionales, el hecho de que se trate de las parejas de mujeres las que más atenúan va en la línea de los resultados de otros estudios, como ocurre, por ejemplo, en las orientaciones narrativas, donde se concluye que las mujeres atenúan más entre ellas justamente por su interés en afianzar los vínculos interpersonales (cf. González Riffo \& Guerrero González 2018). Así, entonces, las conversaciones entre mujeres suelen distanciarse de aquellas en las que participan hombres, probablemente porque, en el afán de generar espacios de armonía interpersonal (Tannen 2007), el carácter afectivo y, en consecuencia, atenuador del diminutivo sirve en esa dirección.

Pese a que varios de los resultados que aquí se han expuesto permiten establecer diálogos con otras investigaciones que se han llevado a cabo sobre esta y otras comunidades habla en relación con el empleo 
del diminutivo, ninguno de los hallazgos presentados, como se evidenció, es estadísticamente significativo, aunque es igualmente interesante revisar con detalle los datos desde el punto de vista descriptivo, ya que podrían conformar tendencias de uso. Sin embargo, para ser consecuentes con lo expuesto, sería factible señalar que no es posible asegurar que exista una distribución variable en el uso de este fenómeno. En este sentido, cabría preguntarse si la idea de que los chilenos utilizan con una alta frecuencia los diminutivos es más bien un fenómeno transversal a los hablantes de esta comunidad de habla, quienes parecen emplear este recurso con una intención afectiva.

\section{Conclusiones}

El objetivo de este estudio fue analizar el uso de diminutivos atenuadores a partir de los métodos de la sociolingüística variacionista. En este contexto, se estableció la frecuencia de uso del recurso y, además, se verificaron las potenciales correlaciones del fenómeno con los factores sexo, nivel de instrucción y tipo de construcción.

En esta línea, ninguno de los resultados que arrojó el análisis fue estadísticamente significativo, por lo que no es posible aseverar que estos resultados representan la realidad de lo que sucede en esta variedad de la lengua española. Aun así, algunos resultados parecen tener un correlato con las descripciones que la literatura ofrece: los hombres atenúan más que las mujeres; los relatos conversacionales por dos mujeres son más atenuados que los que son relatados por dos hombres o parejas mixtas; y en 
las narraciones individuales también se observa más la protección de la propia imagen que en las historias conversacionales.

Desde otra mirada, la imposibilidad de corroborar estos resultados por medio de datos estadísticamente significativos obliga a reconsiderar la posibilidad de que, si se trata de un recurso altamente frecuente en la comunidad de habla, una consecuencia de aquello podría ser que no existiese variación en su uso. Ahora bien, para sostener esta idea habría que estudiar particularmente si la frecuencia de empleo es efectivamente alta en comparación, por ejemplo, con otras variedades de la lengua española.

De la misma forma, se insiste en que revisar el cariz atenuador de los diminutivos no permite en ningún caso describir el fenómeno de la atenuación en términos generales ni mucho menos aseverar que estos datos se repliquen si se estudian los demás recursos de atenuación. En esta línea, es necesario estudiar la posibilidad de que exista variación en cuanto al uso de atenuadores, tanto en narraciones como en otras secuencias discursivas, así como también en producciones escritas.

REFERENCIAS

Albelda, Marta \& Briz, Antonio \& Cestero, Ana \& Kotwica, Dorota \& Villalba, Cristina. 2014. Ficha metodológica para el análisis pragmático de la atenuación en corpus discursivos del español (es.por.atenuación). Oralia 17. 7-62. 
Blas Arroyo, José Luis. 2005. Sociolingüistica del español. Desarrollos y perspectivas en el estudio de la lengua española en contexto social. Madrid: Cátedra.

Bouzas Rosende, Paula. 2017. Atenuación en textos informativos de prensa. Normas 7(2). 91-110. Dor: http://dx.doi.org/10.7203/normas.v7i2.11168

Briz Gómez, Antonio. 2007. Para un análisis semántico, pragmático y sociopragmático de la cortesía atenuadora en España y América. Lingüistica Española Actual 29(1). 5-40.

Briz, Antonio \& Albelda, Marta. 2013. Una propuesta teórica y metodológica para el análisis de la atenuación lingüística en español y portugués. La base de un proyecto en común (ES.POR.ATENUACIóN). Onomázein 28. 288-319. Dor: http://dx.doi.org/10.7764/ onomazein.28.21

Brown, Penelope \& Levinson, Levinson. 1987. Politeness: some universals in language usage. Cambridge: Cambridge University Press.

Chafe, Wallace. 1985. Linguistic differences produced by differences between speaking and writing. En Olson D., Torrance N. \& Hildyard A. (eds.), Literacy, language and learning: the nature and consequences of reading and writing, 105-123. Cambridge: Cambridge University Press.

Cestero, Ana. 2011. Estudio sociolingüístico de la atenuación en el corpus preseea-Madrid. En Cestero, Ana, Molina, Isabel \& Paredes, Florentino. (comps.), Documentos para el XVI Congreso Internacional de la ALFAL, 1897-1906. Alcalá de Henares: Servicio de Publicaciones de la Universidad de Alcalá. 
Cestero, Ana \& Rodríguez Alfano, Lidia. 2014. Guía de estudios de la atenuación en corpus PRESEEA. [Fecha de publicación: 12/06 2014].

Disponible en: http://preseea.linguas.net/Metodología.aspx

Cestero, Ana \& Albelda, Marta. 2012. La atenuación lingüística como fenómeno variable. Oralia 15. 77-124.

Estrada, Olga \& Zárate, Griselda. 2017. Atenuación, género e intensificación en el discurso político: las reacciones mediáticas del caso de una legisladora de Nuevo León, México (2015). Normas 7(2). 125138. Dor: https://doi.org/10.7203/normas.v7i2.11170

Goffman, Erving. 1959. The presentation of self in everyday life. Nueva York: Anchor Books.

Goffman, Erving. 1967. Interactional ritual: essays on face-to-face behavior. Nueva York: Pantheon Books.

Goffman, Erving. 1974. Frame analysis: An essay on the organization of experience. Nueva York: Harper and Row.

Goffman, Erving. 1981. Forms of talk. Filadelfia: University of Pennsylvania Press.

González Riffo, Javier. 2017. Estrategias de atenuación en narraciones de experiencia personal de hablantes de Santiago de Chile: un estudio sociopragmático. Santiago de Chile: Universidad de Chile. (Tesis de maestría).

González Riffo, Javier \& Guerrero González, Silvana. 2017. Estrategias de atenuación en narraciones conversacionales. Lengua y Habla 21. 29-44. http://www.redalyc.org/articulo.oa?id=511954894003

González Riffo, Javier \& Guerrero González, Silvana. 2018. Recursos de atenuación en la 'orientación' de narrativas personales orales desde 
una perspectiva sociopragmática. Nueva revista del Pacífico 68. 62-81. DoI: http://dx.doi.org/10.4067/S0719-51762018000100062.

Gosselin, Laurent. 2010. Les modalités en français. Ámsterdam/Nueva York: Rodopi.

Goodwin, Charles. 1981. Conversational organization: Interaction between speakers and hearers. Nueva York: Academic Press.

Guerrero González, Silvana. 2014. Variación discursiva en narraciones de experiencia personal en el español hablado en Santiago de Chile. Chile: Pontificia Universidad Católica de Chile. (Tesis de doctorado) https://repositorio.uc.cl/handle/11534/22032

Guerrero González, Silvana. 2017. Narración individual versus narración conversacional: un puente entre el variacionismo y la interacción. Onomázein 38(4). 58-87. Dor: https://doi.org/10.7764/ onomazein.38.04

Gumperz, John. 1971. Language in social groups. Essays selected and introduced by A.S. Dil. Stanford: Stanford University Press.

Gumperz, John. 1982. Discourse strategies. Cambridge: Cambridge University Press. DoI: https://doi.org/10.1017/CBO9780511611834

Gumperz, John. 1999. On interactional sociolinguistic method. En Sarangi, S. \& Roberts, C. (eds.) Talk, work and institutional order: discourse in medical, mediation and management settings, 453-471. Berlín: De Gruyter. Dor: https://doi.org/10.1515/9783110208375.4.453

Gumperz, John. 2001. Interactional Sociolinguistics: a personal perspective. En Schiffrin, D., Tannen, D. \& Hamilton, H. (eds.), The handbook of discourse analysis, 215-228. Malden: Blackwell. 
Halliday, Michael. 1985. An introduction to functional grammar. Londres: Edward Arnold.

Haverkate, Henk. 1994. La cortesía verbal. Estudio pragmalingüistico. Madrid: Gredos.

Hernández Campoy, Juan Manuel \& Almeida, Manuel. 2005. Metodología de la investigación sociolingüistica. Málaga: Editorial Comares. Hummel, Martin. 1997. Para la lingüística de vuestro diminutivo: los diminutivos como apreciativos. Anuario de Estudios Filológicos 20. 191-210.

Infante, José María \& Flores, María Eugenia. 2017. Los que dicen sí sin decir sí. Una aproximación a la atenuación y sus recursos en el discurso de políticos mexicanos. Normas. Revista de Estudios Lingüisticos Hispánicos 7(2). 72-90. DOI: 10.7203/normas.v7i2.9595

Labov, William. 1972. Sociolinguistic patterns. Filadelfia: University of Pennsylvania Press.

Labov, William. 2013. The language of life and death: The transformation of experience in oral narrative. Cambridge: Cambridge University Press.

Labov, William \& Waletzky, Joshua. 1967. Narrative analysis: oral versions of personal experience. En Helm, Jane (ed.), Essays on the verbal and visual arts. Proceedings of the annual spring meeting of the American Ethnological Society, 12-44. Seattle: University of Washington Press.

Leech, Geoffrey. 1983. Principles of pragmatics. Londres: Longman. Leech, Geoffrey. 2014. The pragmatics of politeness. Oxford: Oxford. 
López Morales, Humberto. 1994. Métodos de investigación lingüistica. Salamanca: Colegio de España.

Malaver, Irania. 2018. Funciones del diminutivo en el español venezolano. Cuadernos de Lingüistica de El Colegio de México 5(2). 5-44.

Mariottini, Laura. 2006. El uso de diminutivos y su relación con la cortesía lingüística en los chats. Análisis contrastivo de comunidades virtuales españolas e italianas. Cultura, Lenguaje y Representación 3(3). 103-131.

Mendoza, Martha. 2005. Polite diminutives in spanish: a matter of size? En Lakoff R.\& Ide S. (eds.), Broadening the horizon of linguistic politeness. 163-173. Ámsterdam: John Benjamins Publishing.

Merritt, Marilyn. 1982. Distributing and directing attention in primary classrooms. En L. Cherry-Wilkinson (ed.), Communicating in the classroom, 223-244. Nueva York: Academic Press.

Molina Martos, Isabel. 2015. Estrategias de atenuación en el barrio de Salamanca de Madrid. En Cestero, A. M., Molina Martos, I. \& Paredes, F. (eds.), Patrones sociolingüísticos de Madrid, 329-361. Berna: Peter Lang.

Monjour, Alf. 2018. 'Cómo que las 6:45? ¡Si a esa hora es cuando salía el tren!' ‘Se sabe algo de la reunión, era a las 11, no?'. Usos dislocados del imperfecto español como estrategias de atenuación e intensificación. En Albelda, Marta \& Mihatsch, Wiltrud (eds.), Atenuación e intensificación en géneros discursivos, 71-92. Madrid: Iberoamericana Vervuert.

Montecino, Lésmer. 2003. Cortesía y modalización en un foro de opinión en internet. Onomázein 8. 73-98. 
Moreno Fernández, Francisco. 1990. Metodología sociolingüistica. Madrid: Gredos.

Moya, Patricio \& Carrió-Pastor, María Luisa. 2018. La atenuación en los comentarios sobre las noticias digitales en periódicos de España y Chile. Onomázein 40. 56-76. Dor: https://doi.org/10.7764/onomazein.40.04

Norrick, Nick. 2000. Conversational narrative. storytelling in everyday talk. Ámsterdam: John Benjamins Publishing.

Ochs, Elinor. 2004. Narrative lessons. En Duranti, Alessandro (ed.), $A$ companion to linguistics anthropology, 269-289. Malden: Blackwell. Puga Larraín, Juana. 1997. La atenuación en el castellano de Chile. Un enfoque pragmalingüistico. Valencia: Universidad de Valencia. (Tesis doctoral).

Errázuriz L., María José. 2014. [Entrevista a] Juana Puga: Las razones de por qué los chilenos hablan con evasivas. Emol. http://www.emol. com/noticias/Tendencias/2014/03/20/740102/Juana-Puga-Las-razones-de-por-que-los-chilenos-hablan-con-evasivas-y-en-chiquitito. html (Entrevista consultada el 7 de octubre de 2019).

Prieto, Luis. 1995-1996. Análisis sociolingüístico del dequeísmo en el habla de Santiago de Chile. Boletín de Filología xxxv. 379-452.

PRESEEA. 2014-. Corpus del Proyecto para el estudio sociolingüístico del español de España y de América. Alcalá de Henares: Universidad de Alcalá. http://preseea.linguas.net

R Core Team. 2015. R: A language and environment for statistical computing. Vienna: R Foundation for Statistical Computing. 
Real Academia Española y Asociación de Academias de la Lengua Española. 2010. Manual de la Nueva Gramática de la Lengua Española. Madrid: Espasa.

Rodríguez Alfano, Lidia. 2018. La atenuación en justificaciones argumentativas en el corpus Monterrey-Preseea. En Anuario de Letras. Lingüistica y Filología 6(1), 219-250. Dor: http://dx.doi.org/10.19130/ iifl.adel.6.1.2018.1482

Tannen, Deborah. 2007. Talking voices. Repetition, dialogue, and imagery in conversational discourse. Cambridge: Cambridge University Press. Thornborrow, Joanna. 2012. Narrative Analysis. En Gee, J. \& Handford, M. (eds.), The Routledge handbook of discourse analysis. Routledge handbook in applied linguistics, 51-65. Londres: Routledge.

Navarrete, Camila. 2014. ¿Por qué los chilenos hablamos en 'chiquitito’? Entrevista de Julio César Rodríguez a Héctor Veliz para el programa Podría ser peor. https://www.biobiochile.cl/noticias/2014/03/24/ por-que-los-chilenos-hablamos-en-chiquitito.shtml (Consultado 7 de octubre 2019).

Villalba Ibáńez, Cristina. 2018. Atenuación: algunas claves metodológicas para su análisis. Normas 8(1). 306-316. Dor: https://doi. org/10.7203/Normas.v8i1.13277 